\title{
Naturalistic Communication Training for Early Intervention Providers and Latinx Parents of Children with Signs of Autism
}

\author{
Cindy Gevarter ${ }^{1}$ (D) Adriana Medina Najar ${ }^{2}$. Jennifer Flake ${ }^{1} \cdot$ \\ Felicia Tapia-Alvidrez ${ }^{3}$ - Alixandria Lucero $^{3}$
}

Accepted: 16 March 2021/ Published online: 28 April 2021

(C) The Author(s), under exclusive licence to Springer Science+Business Media, LLC, part of Springer Nature 2021

\begin{abstract}
In this study, researchers implemented a brief training plus coaching program in naturalistic developmental behavioral intervention with three participant triads. Each triad consisted of an early intervention provider, an English-speaking Latinx parent, and that parent's young child with autism spectrum disorder (ASD) or early signs of ASD who had limited vocal speech. The effects a single training session, plus two researcher coaching sessions were evaluated using a nonconcurrent multiple probes across participants design. Primary dependent variables included (a) the number of completed targeted communication turns between the parent and child and (b) the number of child independent target communication responses (gestures and manual signs) during family-selected routines. Additional measures examined whether parents used strategies taught to them during training, and whether early intervention providers addressed strategies taught via coaching. A social validity measure was used to determine parent and provider views of the training. Due to COVID-19 restrictions, training and post-training sessions were delivered via telehealth for two triads. While data trends and variability differed across triads, following training, all three families increased the number of completed target communication turns and all three children showed higher rates of independent communication responses. Parents and providers implemented strategies taught and reported positive effects of the program. Implications regarding the use of naturalistic intervention methods for Latinx families, the utility of brief training models to meet the needs of under-resourced early intervention programs, and potential uses of telehealth are discussed.
\end{abstract}

Keywords Early intervention - Autism Spectrum disorder · Naturalistic developmental behavioral intervention $\cdot$ Latino $\cdot$ Telehealth $\cdot$ COVID-19

Cindy Gevarter

cgevarter@unm.edu

Extended author information available on the last page of the article 
Autism spectrum disorder (ASD) is a developmental disability, characterized by deficits in social communication and the presence of repetitive or restrictive behaviors, that is estimated to effect 1 in 54 children (Maenner et al., 2020). Around 25-35\% of young children with ASD may be minimally vocal (Rose et al., 2016). While there are a variety of intervention approaches that may increase the social communication skills of children with ASD, recent meta-analyses provide support for naturalistic developmental behavioral interventions or NDBIs (Sandback et al., 2020a, 2020b). NDBIs embed strategies derived from applied behavior analysis (ABA) and social interactionist/ relational theories within the contexts of everyday routines (e.g., play, mealtime) in natural environments like home, school, and community (Dubin \& Lieberman-Betz, 2020; Schreibman et al., 2015). NDBIs, along with traditional behavioral interventions and developmental interventions, have been shown to have positive effects on language outcomes for children with ASD (Sandback et al., 2020a). Although minimally vocal children may be less likely to make language gains than those with greater vocal skills, it is unclear if a particular intervention approach is best-suited to children with limited vocal skills (Sandback et al., 2020a).

Minimally vocal children may show delays in the development of prelinguistic gestures (e.g., pointing to request or comment, giving items to request assistance, placing arms in air to request up) used to communicate (Shumway \& Wetherby, 2009). When children do not initiate or respond to opportunities to communicate with gestures or other prelinguistic forms (e.g., eye gaze, vocalizations), caregivers may, inturn, limit the quantity and quality of interactions that promote further language and social development (Dubin \& Lieberman-Betz, 2020). A recent study found that during the 9 to 15-month developmental period, when gestures emerge, children with ASD were less likely than peers without disabilities to experience an increase in caregiverchild conversational turns (Swanson et al., 2019). Experiencing more conversational turns, and hearing more adult words at 9-15 months were both correlated with better language outcomes at 24 months (Swanson et al., 2019). Although there are limitations of correlation research, such findings may suggest that increasing prelinguistic communication turns with caregivers may be an appropriate starting point for minimally vocal children.

Prelinguistic skills have been targeted in studies utilizing NDBI components. For instance, a recent review of naturalistic prelinguistic interventions for children with ASD found that a majority of studies with positive effects included NDBI components such as (a) instruction embedded into routines/child-directed activities, (b) systematic prompting (e.g., models, gestures, physical prompts) and time delay (wait time), (c) natural consequences for communication, and (d) environmental arrangements (Dubin $\&$ Lieberman-Betz, 2020). Environmental arrangements increase the number of communicative turns between children and caregivers by structuring routines and materials so that children have to communicate or interact with an adult (Schreibman et al., 2015). Despite evidence supporting the use of NDBI strategies, research is needed to explore effects across cultural groups. For instance, only 6 of the 25 studies reviewed by Dubin and Lieberman-Betz (2020) reported the inclusion of Hispanic children. In the United States, demographic changes suggest a need for more research examining ASD early intervention approaches that meet the needs of Hispanic or Latinx families (Buzhardt et al., 2016; Dubay, 2018). Such research may be particularly applicable in states such as New Mexico, where $69 \%$ of children ages $0-2$ receiving state run early 
intervention services in 2017 were Hispanic or Latinx (United States Department of Education, 2020). Latinx families may be more likely to access state-run early intervention programs rather than more intensive autism-specific services due to culturallinguistic or economic barriers, mismatches between intensive services and culturalfamilial values, and the lack of specialty services in Latinx communities (Magaña et al., 2013, 2016). The state-run early intervention program in New Mexico does not include intensive autism-specific services, but NDBIs can be incorporated into less intensive community programs (Schreibman et al., 2015). The ability to center intervention on family routines may also make naturalistic approaches a good fit for Latinx families (DuBay, 2018).

A recent survey found that Spanish-speaking Latinx parents of children with ASD endorsed several NDBI components (DuBay, 2018). For example, modeling and physical prompting were rated very positively by parents, and following a child's lead and responding to communication were also viewed as helpful. Wait time (i.e., time delay) and environmental arrangements were perceived as more difficult due to concerns they may lead to child frustration. However, parents reported being willing to try such strategies if they felt they would provide long-term benefits (DuBay, 2018). Parents also reported wanting to be involved in intervention, and wanting support from clinicians regarding methods for working with their child at home.

Unfortunately, findings from Dubin and Lieberman-Betz (2020) suggest that parents are not frequently included in naturalistic prelinguistic intervention research. While involving parents in intervention may lead to better generalization for children and positive parental outcomes (Schreibman et al., 2015), Sandback et al. (2020) reported that interventions involving parents alone were less effective for improving language outcomes that clinician-led interventions or interventions involving both parents and clinicians. Traditionally, federally and state-funded early intervention programs make use of coaching models which involve both parents and clinicians. Early intervention coaching involves components such as (a) modeling and discussing target communication behaviors and strategies, (b) observing caregiver-child interactions during routines, (c) assisting caregivers with strategies, (d) providing praise and corrective feedback, and (e) allowing parents to reflect and ask questions (Friedman et al., 2012; Meadan et al., 2020). Coaching can be done in person or via telehealth.

Specialized therapists, such as speech-language pathologists (SLPs) or behavior analysts. May have the knowledge to coach parents in the use of NDBI techniques. However, in New Mexico's early intervention program, there are shortages of specialized service providers (Office of Special Education Programs, 2020). Instead, developmental specialists, who are early intervention generalists with varying educational backgrounds, provide more regular services to families. A recent survey found that New Mexico developmental specialists' knowledge of evidence-based strategies for ASD was significantly lower than SLPs, and the majority of developmental specialists had a knowledge gap in the area of naturalistic intervention (Author et al., 2018). Developmental specialists also reported that working with parents was a primary barrier to serving children with ASD, and noted that they desired training in parent collaboration.

While early intervention studies involving collaboration between community-based providers and parents of children with ASD are limited, (Zwaigenbaum et al., 2015), studies providing training to both providers and parents are even more scarce. In one 
study, Meadan et al. (2020) used a cascading (i.e., train the trainer) telehealth model to teach early intervention providers to coach families of children with developmental disabilities in the use of naturalistic intervention strategies. While providers increased their use of coaching strategies, participating families did not specifically include children with characteristics of ASD, and the study did not evaluate effects on parents' use of strategies or children's communication behaviors.

In under-resourced states like New Mexico, examining the effects of brief trainings involving both parents and early intervention providers may have real-world applicability. For instance, if SLPs or behavior analysts with knowledge of NDBI approaches could provide consultative training and coaching to developmental specialists and families (in-situ or via telehealth), developmental specialists could continue to coach families during weekly visits. Exploring such approaches with Latinx families is critical in New Mexico and other states. Thus, the primary purpose of the current study was to determine the effects of a brief training/coaching program (focused on NDBI strategies) for developmental specialists and Latinx families with children with ASD or early signs of ASD. As the completion of turns using early communication forms (e.g., gestures) may be related to the development of further language skills (Dubin \& Lieberman-Betz, 2020; Swanson et al., 2019) the primary outcome measures in this study were the number of completed target communication turns between a parent and child, and the number of child independent target communication responses. Although the training/ coaching program was designed to be implemented in-situ, following orders to prevent the spread of COVID-19, methods were modified to allow for training/coaching, and post-training sessions to occur via telehealth for two of the three families. The primary research question was as follows:

What were the effects of a brief training/coaching program (delivered in-situ or via telehealth), that taught NDBI strategies to Latinx parents and early intervention providers (i.e., developmental specialists), on (a) the number of completed targeted communication turns between a parent and child with ASD or signs of $\mathrm{ASD}$, and (b) the number of child independent target communication responses during natural routines?

Secondary research aims focused on describing (a) how often parents employed NDBI strategies, (b) which NDBI strategies developmental specialists addressed with parents during coaching sessions, and (c) parent and provider views on the social validity of the training.

\section{Methods}

\section{Participants}

The study was approved by an institutional review board. Participant triads (developmental specialist, parent, and child) were recruited from a New Mexico agency that provides free early intervention (birth-three) services to families from low-income backgrounds living in or around a mid-sized city. A majority of families served are Latinx. The lead researcher asked the agency's staff to recommend a pilot family 
receiving services from a developmental specialist that met the following criteria (a) at least one parent was proficient in English, (b) child receiving services had an ASD diagnosis, or screened as high risk for ASD (scores 8-20) on the Modified Checklist for Autism in Toddlers Revised with Follow-up (M-CHAT-R/F), and (c) child had minimal vocal speech (less than 15 words). The M-CHAT-R/F is a validated autism screener for children ages 16-30 months (Robins et al., 2014) that is administered to all children in New Mexico's early intervention program. A diagnosis of ASD was not used for inclusion due to long waitlists for ASD assessment in New Mexico. Supervisors recommended a family, and after obtaining informed consent, a pilot study was conducted. Following the pilot, the researcher asked supervisors to recommend three additional families who met criteria. The researcher met with each provider and parent to obtain informed consent. Parents consented for children's participation, and measures of child-assent were described.

Table 1 provides demographic and assessment information for the three participating triads. Ethnicity was self-reported. All child participants were Latinx males between 1.9 and 2.9 years-old. Children 1 and 2 scored as high risk for ASD on the M-CHAT-R/F and were on evaluation waitlists. Child 3 had an ASD diagnosis. Parents 1 and 2 were Latinx fathers. Parent 3 was a Latinx mother with a learning disability. Parent 1 was bilingual (English/Spanish), while Parents 2 and 3 primarily spoke English. Developmental specialists were female (Caucasian, Latinx, and bi-racial). The first developmental specialist for Triad 3 quit the agency week 3 of baseline. The provider who took over (a Latinx female) consented to participate.

\section{Child Communication Targets}

Based on parent report using the Communication Matrix (Rowland, 2011; see materials and setting), all three children primarily used unconventional gestures to communicate, with some emerging conventional gestures. A manual sign for MORE had been introduced to children 2 and 3, but neither used it independently. Child 3 used about five vocal approximations/or sound effects (e.g., uh-oh). Using information collected during baseline (see procedures) three target responses were selected for each child. Child 1's targets included pointing to request new items, using an idiosyncratic gesture to request an action (Go), and giving items to request assistance. Child 1's parents reported that pointing and giving items occurred on occasion, but not consistently. No targets were observed during baseline. Targets for children 2 and 3 included signing MORE to request more of an action or item, signing OPEN to request assistance, and pointing to make choices or request new items. Both sets of parents/providers had begun introducing signs and both families indicated that this was a preferred modality. Children 2 and 3 were each observed to imitate MORE during one of their baseline probes, but opportunities were limited. Neither family had introduced OPEN, but during baseline both parents frequently opened objects of interest (e.g., books, bags of toys). Child 2 used pointing to comment during baseline (e.g., pointing to items in book), but not to make choices or request (which his parents reported as an area of concern). Child 3 did not point or reach to request during baseline, but his mother reported that he pointed on occasion. His pointing target was modified to include either pointing or reaching, as 


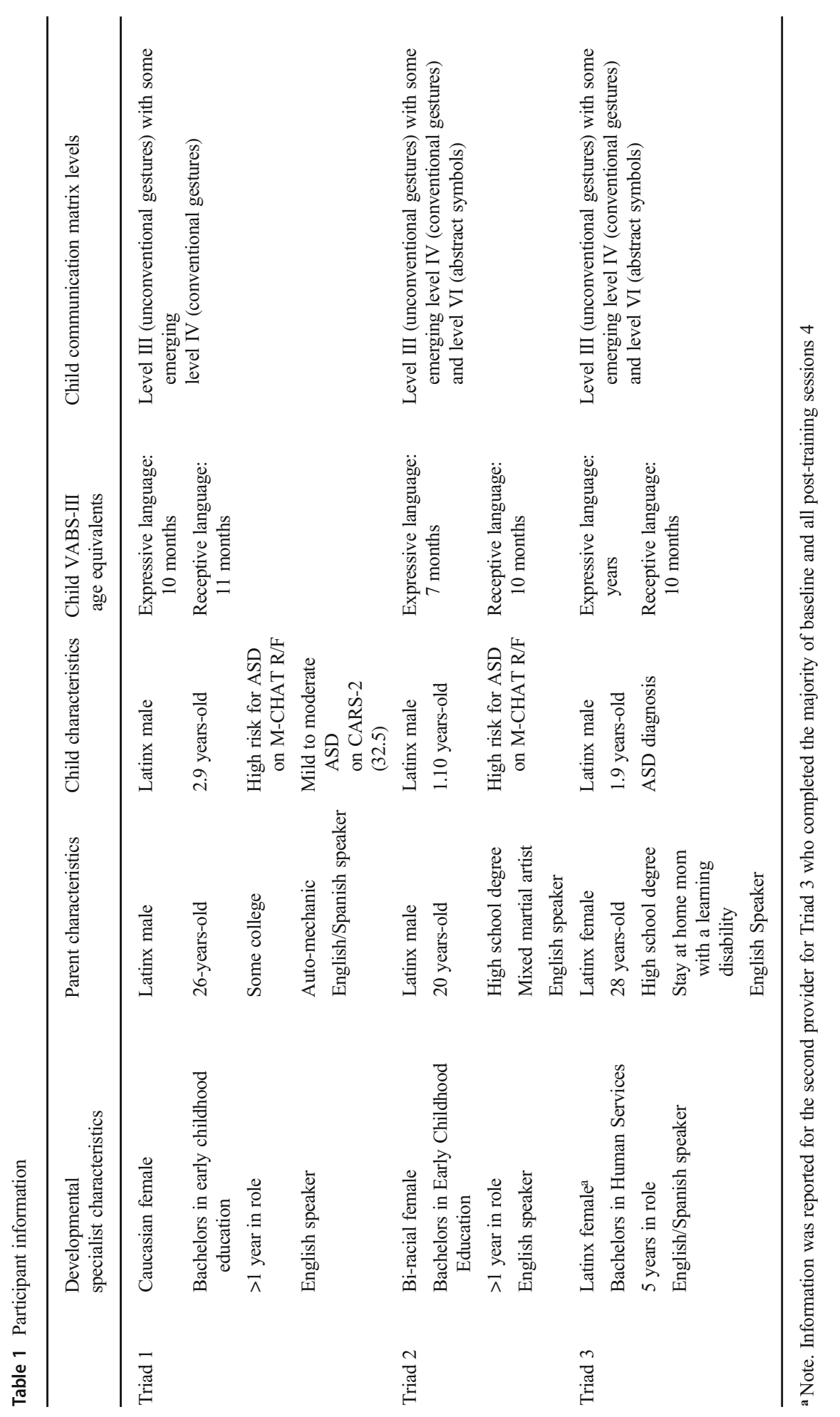


he showed an aversion to his mother physically prompting his finger to point to certain objects.

\section{Materials and Setting}

Walmart gift cards were used as incentives. Developmental specialists received a $\$ 50$ gift card per session and parents received $\$ 25$. The researchers also used commercially available assessments including the Childhood Autism Rating Scale Second Edition (CARS-2; Schopler et al., 2010), the Vineland Adaptive Behavior Scale (VABS-III) 3rd Edition (Sparrow et al., 2016) and the Communication Matrix (Rowland, 2011). The CARS-2 is used to describe the severity of ASD symptoms for children ages two and above, and classifications correlate with other measures (Reszka et al., 2014). CARS-2 severity levels were determined for participants older than two. Communication sections of the VABs-III were administered for all participants. The VABS-III has strong internal consistency and indicators of validity (Pepperdine et al., 2018). The Communication Matrix provides an assessment of a child's communication skills using a developmental approach that is simple to administer and is widely used (Rowland, 2011).

All sessions took place in families' homes. Parents were asked to use preferred materials (e.g., toys, activity-based items, foods, drinks) that they would normally use to engage their child during everyday activities. Examples included bubbles, balloons, blocks, books, cars, a mower, figurines, sippy cups, and fruit snacks. The researchers did not restrict item selection, but provided suggestions regarding items/activities that appeared to be most engaging after baseline.

Training materials involved video models and visual supports. Video models included three separate training videos that ranged from 2 to 8 mins in length. In the baseline training video the lead researcher interacted with a 3-year-old boy with ASD (not a study participant) by playing with the child and providing verbal descriptions of his play (e.g., labeling items he interacted with), but did not use any other NDBI strategies. In the intervention training video the researcher continued to verbally comment on play, and modeled the following strategies with the child: (a) creating and capturing communication opportunities (via environmental arrangements and following the child's lead), (b) waiting for a response, (c) prompting with models and partial or full physical prompts, (d) responding to communication by giving items and doing actions, and (e) pairing vocal models related to targeted communication responses (e.g., saying up when child put arms in air). Methods for creating and capturing opportunities included giving the child items that required assistance, using items with multiple pieces, starting and then stopping a routine (like picking child up) to require a response, placing items slightly out of reach (e.g., held up or on shelf), following the child's lead (e.g., offering items the child walked towards), and taking turns with/modeling how to play with items. For waiting/prompting, the video provided examples of when to wait longer and use model prompts for partially known skills, or wait less and consider physical prompts for lesser-known skills. The video also reviewed ways to increase play engagement (e.g., model a funny or different way to play). A visual support that listed the strategies described in the video was created. Finally, in a coaching video the researcher coached the model child's mother to use the strategies from the previous video by providing direct assistance (e.g., helping to 
prompt the child, or handing the parent items to use for a communication opportunity), giving verbal suggestions or feedback, and praising strategy use. A visual support describing these coaching methods was created. A blank activity planner was also created for training. The planner allowed participants and the researcher to collectively fill in target communication behaviors, potential activities/items that may help elicit those behaviors, strategies to create/capture opportunities, and how to wait/prompt those behaviors.

\section{Dependent Variables}

\section{Primary Measures}

The primary dependent variables were the number of completed targeted communication turns between the parent and child, and the number of child independent target communication responses. To be considered a completed targeted turn, the following criteria had to be met (a) the parent was involved in creating or capturing an opportunity for the child to use a target communication response, (b) the child made either a prompted or independent target response, and (c) the parent responded by giving items or completing actions requested by the child. The target responses selected for each child (see participants) were collaboratively determined during training (see procedures). Definitions describing the form and function of each child's target responses were created. Child independent target communication responses were those that did not involve any model, gesture, or physical prompts.

\section{Strategies Used by Parents}

Three measures provided information about parents' use of NDBI strategies taught during training. First, the total number of turns (complete and incomplete) indicated how often parents created or captured communication opportunities. The percentage of completed targeted turns (completed turns divided by total turns) provided a measure of how often parents followed through with a communication opportunity by (a) prompting communication as needed, and (b) responding to communication. Finally, parents' use of verbal pairing was determined by calculating the percentage of targeted turns in which the parent used a vocal model related to the target (e.g., saying open after child signed OPEN).

Strategies Addressed by Providers Initially, the researchers planned to measure how often developmental specialists used direct assistance, verbal suggestions/feedback, or praise to coach parents in the implementation of (a) creating/capturing opportunities, (b) waiting (c) prompting, (d) responding, or (e) verbal pairing, during post-training sessions without the researcher. After transitioning to telehealth, direct assistance was no longer possible and it appeared that verbal suggestions/feedback were given less intermittently. Instead, providers gave feedback/suggestions only after parents' speech/ interactions had clearly paused for a period of time (likely impacted by the nature of Zoom). Thus, rather than use a frequency measure to examine how often each strategy was addressed, and with what coaching approach (direct assistance, verbal suggestions/ feedback, or praise), the researchers created a binary addressed/not addressed measure 
to indicate whether the developmental specialist provided coaching in creating opportunities, waiting, prompting, responding or verbal pairing, with ANY of the coaching methods (i.e., direct assistance for Triad 1 only, or via verbal suggestions/feedback, or praise for all Triads) during a session. A binary used/not used code for general praise of parents' overall use of strategies (i.e., praising implementation of a full sequence of create, wait, prompt and respond rather than giving praise for a discrete strategy) was also created.

\section{Data Collection}

\section{Coding of Primary Dependent Variables and Parent Strategy Use}

All sessions were videotaped and independent observers (an undergraduate and graduate student) used a session data sheet to record the response components of the primary dependent measures and parent strategy use measures. Specifically, whenever a parent was involved in creating or capturing an opportunity for a targeted communication turn, coders recorded (a) whether the child responded independently, prompted, or not at all, (b) whether the parent responded to the child's communication, and (c) whether the parent provided a vocal model related to the target. Components $\mathrm{a} / \mathrm{b}$ were used to mark whether a communicative turn was complete. For each session, coders then indicated the number of (a) completed target communication turns between the parent and child, (b) the number of independent child target communication responses, (c) the total number of target turns (complete turns + incomplete turns), (d) the percentage of total turns that were completed, and (e) the percentage of total turns with parent vocal modeling.

\section{Coding for Strategies Addressed by Providers}

Trained observers watched all post-training videos that involved the provider and family alone and used a checklist to mark whether the provider specifically addressed created opportunities, waiting, prompting, responding, verbal pairing (using any of the coaching methods) or gave praise for general use of strategies. Observers received training in the definition of each communication and coaching strategy.

\section{Inter-Observer Agreement (IOA)}

For each triad, $33 \%$ of sessions (including sessions across all phases) were randomly selected for IOA checks. The graduate and undergraduate students who served as the primary coders completed IOA checks for triads for which they were not the primary coder. Instances in which one coder included an opportunity that a second did not, marked the child's response differently than the second coder, or marked the occurrence of a parent response differently than the second coder, were all disagreements. IOA was determined by dividing the total agreements by (disagreements + agreements). Mean IOA scores were 93\% (range 86-100\%) for Triad 1, 86\% (range 75$100 \%$ ) for Triad 2, and 92\% (range 83-100\%) for Triad 3. IOA for parent vocal pairing was also conducted on the same sessions. Mean scores were $94 \%$ (range 86-100\%) for Triad 1, 91\% (77-100\%) for Triad 2, and 92\% (88-100\%) for Triad 3. Finally, IOA for 
providers' use of strategies was conducted for at least $33 \%$ of coded sessions. Independent coders used the same checklist as the primary coders to mark what strategies were addressed during sessions. Mean IOA scores were $83 \%$ for Triads 1 and 2, and 92\% (range $83-100 \%$ ) for Triad 3.

\section{Research Design}

The study utilized a non-concurrent multiple-probes across-participants design (Watson \& Workman, 1981; Harvey et al., 2004). Research sessions occurred twice a week during early intervention provider visits. Each research session was $10 \mathrm{~min}$ long. When a given triad became available, they were assigned to pre-determined baseline lengths of 2 weeks with 4 probes (Triad 1), 3 weeks with 5 probes (Triad 2), and 5 weeks with 6 probes (Triad 3). Assignments were not be completely random due to circumstantial issues. For instance, Triad 1 was assigned to the shortest baseline length due to the fact that he was going to be aging out of early intervention soon. The provider working with Triad 3 quit the agency after week 3 of baseline. While a new provider was able to take over during week 4 , the researchers did not conduct probes this week to allow time for the provider and family to build rapport. Two more probes were conducted with the newly assigned provider during weeks 5 and 6 (sessions 9 and 11). A final probe to be conducted on session 12 was cancelled due to COVID-19 restrictions.

\section{Procedures}

\section{Community Collaboration and Pilot}

Following a literature review of naturalistic interventions for prelinguistic communication, the first three authors created an outline of potential intervention strategies. The lead researcher met with the early intervention program director and two supervisors to discuss ideas. The community team members endorsed the NDBI strategies selected and noted that emphasizing the role of the provider as a coach, rather than primary communication partner, would be helpful. Using feedback, the first three authors developed a training that was implemented with a pilot triad. Following the pilot, the lead researcher met with the director and supervisors to discuss changes. Changes focused on including both the provider and parent in the initial training, adding video models, and including two follow-up coaching sessions with the researcher.

\section{Screening}

During screening, the researcher met with the parent and provider and administered the VABS-III and the Communication Matrix (and the CARS-2 for child 1). Parents were also asked to select a common routine that would occur during scheduled sessions. All three parents selected play, but noted that it was common for children to also have snacks/drinks items during play routines. Parent 2 also noted that they frequently interspersed storybook reading with play. 


\section{Baseline}

During baseline probes, parents were asked to engage with their child as they normally would during play routines, using any materials or activities that they would naturally select. Providers were instructed to do what they would normally do during an intervention session. Once a child showed interest in any items, materials, or activities offered by a parent or provider, a timer was started and 10-min samples of the routine were recorded. During baseline, one probe in which the provider was not present was also conducted. Initially, this probe was conceptualized as a measure of generalization across people (i.e., generalizing skills from contexts with the parent, provider and child interacting, to contexts with only the child and parent). However, given that providers 2 and 3 were not physically present during post-training sessions, these probes were reconceptualized as probes without provider coaching.

\section{Training}

Prior to training, the first three authors reviewed each child's Communication Matrix profile and watched baseline videos in order to create a list of possible communication targets. This list was created in consideration of absent or emerging communicative functions/forms (e.g., child reaching to make choices, but not pointing) the types of interactions, items, and activities in which the child showed interest during baseline (e.g., child engaging in movement-based activities), and the potential for communication opportunities (e.g., a parent pushing a child in a car while modeling Go, but not waiting for a response). Following baseline, the lead researcher then conducted the training session with the parent and provider. A second, non-participating parent, was also present for Triads 1 and 2. For Triad 1 (conducted in-situ) a research assistant was available to engage the target child. The training lasted about $1-1.5 \mathrm{~h}$.

In order to provide a clear rationale for developing early communicative behaviors, the researcher first reviewed the Communication Matrix and described where the target child was currently functioning. Next, to provide a rationale for strategies, the researcher played the baseline training video in which the lead researcher did not use strategies with a model child. The researcher described how even though she was talking a lot in the video, the child was not taking communicative turns. Next, the researcher played the intervention training video (see materials for a description of strategies modeled) and discussed how the child was now taking communicative turns. The researcher provided parents and providers with the visual support that outlined the naturalistic strategies, and provided an opportunity for questions. Next, the researcher played the coaching video and introduced the accompanying coaching visual support. After reviewing training videos and visuals, the researcher played example clips from the target child's baseline sessions during which a communicative turn could have been created or completed (e.g., parent saying do you want more? but then giving the child items without requiring a response). The researcher then guided the team to collaboratively select three target communication behaviors, providing suggestions from the list generated by the first three authors, and eliciting suggestions for forms and functions that were preferred, culturally-relevant, and family-appropriate (see participants section for a descriptions of targets). Children's existing abilities were also taken into account (e.g., whether child had been observed or reported to demonstrate use of gestures or signs similar in form to 
those selected). After agreeing upon targets, the researcher, provider, and parents collaboratively filled out the activity planner (see materials). Next, the researcher modeled selected strategies with the target child and the parents and provider were given opportunities to practice, receive feedback and ask questions.

Modifications for Telehealth Training Due to COVID-19 restrictions, the training was adapted to occur over a live video technology (Zoom) for Triads 2 and 3 . The primary elements of the training remained the same, with the researcher using screen sharing to review all videos and materials. Materials were also emailed to the parents and provider. The same coaching video and coaching visuals were used, however, the researcher discussed that although the provider could no longer directly assist with strategy implementation, the provider could use verbal feedback/suggestions and praise. Researcher modeling with the target child could not be conducted, but parents could practice strategies, receive feedback, and ask questions.

\section{Post-Training Sessions}

During post-training sessions, parents and providers were asked to implement routines using the intervention strategies/coaching methods introduced during training. These sessions were also $10 \mathrm{~min}$ in length and began when the child showed interest in and item or activity presented by the parent or provider. During the 1st and 3rd post-training sessions, the researcher was present or available via Zoom (triads 2-3) to provide additional coaching to the parent and provider. At the start of the first post-training session, the lead researcher asked if anyone wanted to review targets/strategies or had any questions. The parent and developmental specialist then began the 10-min session and the lead researcher provided suggestions, feedback, and praise to both the parent and provider. For Triad 1, the researcher also modeled or assisted with strategies as needed. At the end of the session, the researcher reviewed suggestions and asked if there were any remaining questions. During the second post-training session, the lead researcher was not present or available via Zoom. On the third post-training session, the researcher was again available to provide feedback, suggestions, and praise. Coaching from the researcher was then completely faded by the fourth posttraining session. All families were scheduled to have five additional post-training sessions with developmental specialists, but due to COVID-19, Triad 1 only had three additional sessions. Due to a provider absence, Triad 3 had four sessions.

Sessions without Provider Coaching Three sessions, in which the provider was no longer present or available via Zoom, were conducted to assess whether parents continued to complete communicative turns without coaching, and whether children continued to use independent responses. Due to COVID-19, sessions were recorded remotely for all three families.

\section{Treatment Fidelity}

Checklists with the steps for the primary training session (e.g., go over Communication Matrix, introduce videos etc.), and researcher coaching sessions (e.g., provide 
opportunity for questions, give feedback etc.) were created. Trained independent observers (graduate and undergraduate students) marked off whether the lead researcher completed each step in the checklist. Fidelity was $100 \%$ across all trainings and coaching sessions. Sample checklists are included in supplementary materials.

\section{Data Analysis}

The two primary dependent variables were graphed for each session across phases. Differences in trend, variability, and immediacy of effect were evaluated using visual analysis methods described by Kratochwill et al. (2013). To complement visual analysis, the Nonoverlap of All Pairs (NAP) effect size measure (Parker \& Vannest, 2009) was used. Although there is not one agreed upon effect size measure for single case experimental designs, NAP is a measure with high statistical power that is appropriate when there are no baseline trends (Parker et al., 2011).

NAP scores are calculated by comparing all baseline and intervention points and providing a percentage of all non-overlapping points. NAP scores of .0-.65 indicate weak effects, 0.66-0.92 medium effects, and 0.93-1.0 large or strong effects (Parker \& Vannest, 2009).

\section{Social Validity Survey}

The researchers developed an online social validity survey consisting of eight openended questions (focused on the benefits and challenges of training) that was sent to providers and parents. While many social validity assessments make use of rating scales and yes/no questions, topics important to participants might be overlooked (Leko, 2014). In contrast, open-ended questions may lead to unexpected results that provide direction for further research (Leko, 2014).

The first three authors worked collaboratively to develop open-ended questions that addressed both successes, challenges, and suggestions for improvements. Example questions included "What elements of the training were most useful?" and "What (if any) challenges did you experience?" A copy of the survey is included in supplementary materials.

\section{Results}

\section{Primary Dependent Variables}

Figure 1 displays the number of completed targeted communication turns between the parent and child, and the number of child independent target communication responses across phases and triads. For all triads, there were improvements in both dependent measures following training. NAP scores for completed targeted communication turns were 1.0 for all three triads. Confidence intervals were CI90 $=0.41-1.0 ; p=0.0055$ for Triad 1; CI90 $=0.47-1.0 ; p=0.0018$ for Triad 2, and CI90 $=0.42-1.0 ; p=0.0047$ for Triad 3. NAP scores for child independent target communication responses were 1.0 for Triads 1 and 3 and 0.95 Triad 2. Confidence intervals were CI90 $=0.41-1.0 ; p=$ 


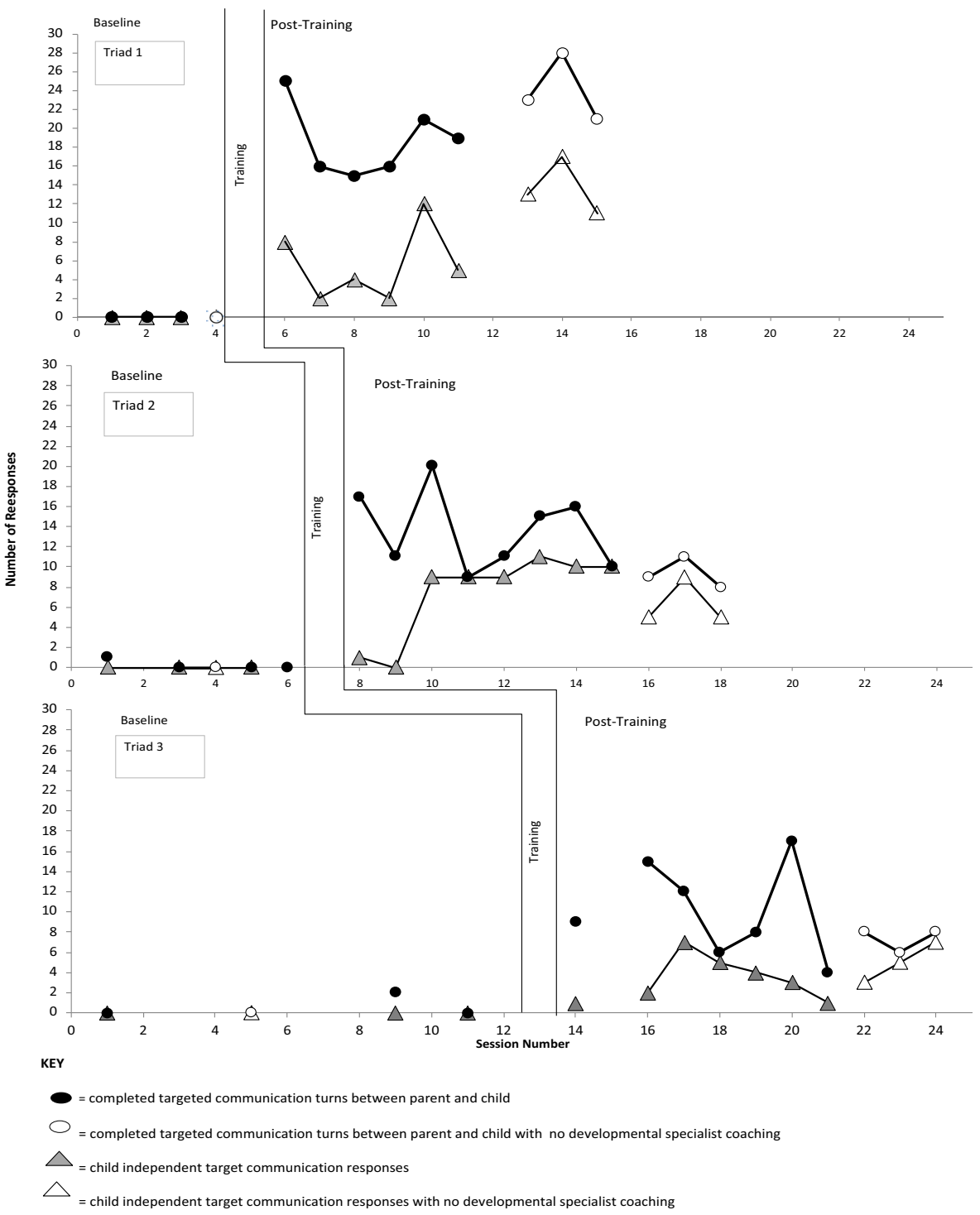

Fig. 1 The number of completed targeted communication turns and the number of child independent target communication responses across phases and triads. Note: researcher coaching was provided on the 1 st and $3 \mathrm{rd}$ session of post-training. Telehealth sessions began during training for Triads 2 and 3 and during post-training sessions without the developmental specialist for Triad 1.

0.0055 for Triad $1 ;$ CI90 $=0.336-1.0 ; p=0.009$ for Triad 2, and CI90 $=0.42-1.0 ; p=$ 0.0047 for Triad 3 .

While NAP scores indicate large effects, visual analysis suggests differences in trend, level, and variability. Triad 1 had an immediate increase in total completed targeted turns during the first post-training session with researcher coaching. Completed targeted turns then decreased slightly, but continued to maintain above baseline at high levels (16-21 turns per session), and increased further when developmental 
specialist support was faded (23-28 turns per session). Similarly, after an immediate increase in independent responding during the first post training session (primarily with one specific target), child 1's independent responses decreased slightly. He did, however, continue to use independent responses at levels higher than baseline during all post-training sessions (with and without the provider) and had an overall increasing trend.

Triad 2 showed an immediate increase in the total completed targeted turns during the first post-training session. The most completed turns occurred during the third posttraining session with researcher coaching (20 completed turns). There was variability in the number of completed turns when researcher coaching was faded, however, rates maintained at levels above baseline during remaining post-training sessions with and without developmental specialist support (8-16 completed turns). Child 2 used one independent response during the first two post-trainings, and increased to eight independent responses by the third session. He maintained high rates of independent responding during remaining post-training sessions with provider support (9-11 responses), and decreased slightly when provider support was faded (5-9 responses).

Triad 3 also showed an immediate increase in the total completed targeted turns during the first post-training session. There was high variability in the number of completed turns during remaining post-training sessions (4-17 turns per session), but all rates were above baseline. Child 3's independent responding was initially on an upward trend during the first three post-trainings. Although his rates then decreased, he still used at least one independent response per session, and responding began increasing again towards the end of the study.

\section{Strategies Used by Parents}

Table 2 provides the average total target turns (complete and incomplete), the average percentage of completed targeted turns, and the average percentage of turns with parent verbal pairing across phases. All parents had low average rates of total target turns during baseline that increased during post-training, and maintained when provider support was faded. Triad 1 did not complete any turns during baseline, and Triads 2 and 3 had low completion rates. Average completion rates during post-training (with and without provider support) were above $80 \%$ for all triads (with Triad 2 having the most variability). Parent 1 did not use vocal models during baseline, but consistently used verbal pairing during post-training with mean percentages above $90 \%$. Parent 2 used vocal models at variable rates during all phases (with mean percentages ranging from $77 \%$ to $88 \%$ ). Parent 3 inconsistently used vocal models during baseline (mean $50 \%$ ), and although there was variability, mean percentages were above $85 \%$ for posttraining.

\section{Strategies Addressed by Providers}

All providers addressed at least one of the primary intervention strategies during all post-training sessions without the researcher. During their first session without the researcher (post-training 2), all three providers gave general praise and addressed at 
Table 2 Averages across phases for parent strategy use measures

\begin{tabular}{|c|c|c|c|c|}
\hline Triad & Measures & & Phase & \\
\hline \multirow{5}{*}{$\begin{array}{c}\text { Triad } \\
1\end{array}$} & & Baseline & Post training & $\begin{array}{l}\text { sessions without } \\
\text { provider coaching }\end{array}$ \\
\hline & Average total target turns & $M=2($ range $0-6)$ & $M=21$ (range16-28) & $M=26$ (range $23-28$ ) \\
\hline & $\begin{array}{l}\text { Average percentage } \\
\text { of completed targeted turns }\end{array}$ & $\mathrm{M}=0 \%$ & $\begin{array}{l}\mathrm{M}=91 \% \\
\quad(\text { range } 75-100 \%)\end{array}$ & $\begin{array}{l}M=93 \% \\
\quad(\text { range } 89-100 \%)\end{array}$ \\
\hline & $\begin{array}{l}\text { Average percentage } \\
\text { of turns with parent vocal } \\
\text { modeling }\end{array}$ & $\mathrm{M}=0 \%$ & $\begin{array}{l}M=97 \% \\
\quad(\text { range } 90-100 \%)\end{array}$ & $\begin{array}{l}\mathrm{M}=94 \% \\
\quad(\text { range } 93-96 \%)\end{array}$ \\
\hline & & Baseline & Post training & Generalization \\
\hline \multirow{4}{*}{$\begin{array}{c}\text { Triad } \\
2\end{array}$} & Average total target turns & $\mathrm{M}=3$ (range $2-5)$ & $M=15($ range $10-20)$ & $M=12($ range $9-13)$ \\
\hline & $\begin{array}{l}\text { Average percentage } \\
\text { of completed targeted turns }\end{array}$ & $\begin{array}{l}M=13 \% \\
\quad(\text { range } 0-50 \%)\end{array}$ & $\begin{array}{l}\mathrm{M}=88 \% \\
\quad(\text { range } 73-100 \%)\end{array}$ & $\begin{array}{l}M=82 \% \\
\quad(\text { range } 62-100 \%)\end{array}$ \\
\hline & $\begin{array}{l}\text { Average percentage } \\
\text { of turns with parent vocal } \\
\text { modeling }\end{array}$ & $\begin{array}{l}M=78 \% \\
\quad(\text { range } 40-100 \%)\end{array}$ & $\begin{array}{l}\mathrm{M}=85 \% \\
\quad(\text { range } 54-100 \%)\end{array}$ & $\begin{array}{c}M=81 \% \text { (range } \\
69-89 \%)\end{array}$ \\
\hline & & Baseline & Post training & Generalization \\
\hline \multirow{3}{*}{$\begin{array}{c}\text { Triad } \\
3\end{array}$} & Average total target turns & $M=1($ range $0-5)$ & $\mathrm{M}=11$ (range $4-17)$ & $M=12($ range $8-18)$ \\
\hline & $\begin{array}{l}\text { Average percentage } \\
\text { of completed targeted turns }\end{array}$ & $\begin{array}{l}M=15 \% \\
\quad(\text { range } 0-60 \%)\end{array}$ & $\begin{array}{l}\mathrm{M}=94 \% \\
\quad(\text { range } 80-100 \%)\end{array}$ & $\begin{array}{l}\mathrm{M}=84 \% \\
\quad(\text { range } 75-89 \%)\end{array}$ \\
\hline & $\begin{array}{l}\text { Average percentage } \\
\text { of turns with parent vocal } \\
\text { modeling }\end{array}$ & $\begin{array}{l}M=50 \% \\
\quad(\text { range } 0-100 \%)\end{array}$ & $\begin{array}{l}\mathrm{M}=87 \% \\
\quad(\text { range } 42-100 \%)\end{array}$ & $\begin{array}{l}M=88 \% \\
\quad(\text { range } 75-100 \%)\end{array}$ \\
\hline
\end{tabular}

least four of the five main strategies. The number of strategies addressed decreased for following sessions. Provider 1, who completed in-situ sessions, continued to address at least three strategies per session. She addressed prompting, verbal pairing, and creating opportunities during all sessions, occasionally addressed responding, and did not address waiting. Providers 2 and 3 used general praise every session and addressed at least one intervention strategy on all sessions. Provider 2 most often addressed prompting, waiting, and responding, less frequently addressed creating opportunities, and did not address verbal pairing. Provider 3 most often addressed prompting, verbal pairing and creating opportunities, less frequently addressed responding, and did not address waiting.

\section{Social Validity}

All parents and developmental specialists described things that went well during training (e.g., learning new strategies, seeing child's communication increase, having follow-up and feedback) and described ways in which they would continue to apply strategies in other contexts (e.g., parents using strategies across other routines, providers applying methods with other families). All respondents also described improvements in target children's communication skills. For instance, parent 2 noted that his 
child "...used all three signs frequently and fluidly and in the correct context $90 \%$ of the time, whereas before his only communication was whining." Elements of the training described as most useful included researcher-modeled demonstrations of strategies, learning how to create opportunities and play in different ways, and having visual supports describing the strategies. Parents and providers did not report any suggestions to improve training, but two parents noted challenges such as waiting for the child to communicate, and learning to have higher expectations. Two providers noted that they initially experienced feelings of discomfort incorporating a new person in a session and feeling unsure of themselves. The third developmental specialist noted that conducting sessions via video was a challenge.

\section{Discussion}

In this preliminary study, following researcher-led training in NDBI communication strategies (with two follow-up researcher coaching sessions), Latinx parents increased the number of target communication turns they completed with their children during typical family routines with early intervention provider coaching. Parents also continued to complete turns when provider coaching was faded. Child participants with ASD or early signs of ASD increased independent target communication responses. Early intervention providers coached parents in strategies taught during the training, and parents applied strategies with and without provider support. Parents and providers described the intervention positively and noted they would continue to use strategies. Findings suggest implications for clinical practice and limitations provide direction for future research.

\section{Clinical Implications}

This study was designed to meet the "real world" needs of under-resourced community-based early intervention programs. While more intensive training programs delivered by clinicians/researchers to parents alone are common in the research (Trembath et al., 2019), findings from this study demonstrate that brief consultative training and coaching with parents and community-based providers may also lead to positive outcomes. While this study did not measure long-term outcomes or address a wide range of adaptative or communicative skills, there were still immediate changes to parent behavior, and gradual changes to children's communication skills. Notably, these changes involved increases in communicative turns, and children's independent use of early communication behaviors; factors correlated with further development of language and social skills (Medeiros \& Winsler, 2014; Özçalıșkan et al., 2016; Swanson et al., 2019). Although the direct effects of providers' coaching after researcher support was faded cannot be determined, the fact that developmental specialists continued to address intervention strategies is promising. Providers may have appropriately faded the number of strategies they addressed over time as parents became more proficient. Community-based agencies who do not have enough highly-trained therapists (e.g., behavior analysts, SLPs) to deliver direct services to families should consider adapting therapists' roles so that they can provide training and coaching during co-sessions with developmental specialists or other generalists. Therapists could 
design a series of trainings addressing other intervention strategies (e.g., responsive interactions) and provide intermittent follow-up coaching sessions.

In addition to addressing the needs of under-resourced programs, this study supports prior research reporting positive outcomes for telehealth (Sutherland et al., 2018). Although the family who completed training and post-training in-situ had the highest levels of responding, the two triads that participated via telehealth still showed increases in the primary dependent variables. It is unclear whether direct access to the researcher or developmental specialist impacted results, however, Provider 1 was able to consistently assist Parent 1 with prompting, creating opportunities, and reengaging the child. Such direct assistance may have been helpful for Triad 3, as Child 3 was most likely to become disengaged, and Parent 3 (who had a learning disability) experienced some challenges delivering prompts and managing materials. Thus, while telehealth may lead to improvements across parent and child outcomes, practitioners may also consider when, if possible, supplemental in-situ sessions may be appropriate. Prior qualitative research has, in fact, indicated that families of children with ASD who report positive aspects of telehealth, also suggest that they would benefit from some inperson sessions (Ashburner et al., 2016). Telehealth implications extend beyond the pandemic as many under-resourced early intervention agencies are based in rural areas with even greater disparities in access to specialized therapists. The fact that the current training model was easily adapted to telehealth may provide support for hybrid inperson + telehealth models (e.g., where trainer is remote, and provider is present). Future research could compare in-situ, telehealth, and hybrid models.

Finally, findings from this study support prior research indicating that NDBI approaches may be a viable option for Latinx families (DuBay, 2018). All parents in this study indicated that the training went well, that their children made improvements in communication, and that they would continue to use methods across routines. Minimal challenges (e.g., adjusting expectations, waiting for child to communicate) were reported and parents did not have suggestions to improve the training. Acceptance of intervention methods may have been impacted by the rationale building and inclusive team approach that occurred during training. While involving families and community providers in goal selection may help ensure cultural and familial relevancy, adaptations may still be needed. For instance, given prior research indicating that Latinx parents might express concern with strategies that appear to frustrate the child (DuBay, 2018), during coaching sessions the researcher provided suggestions to reduce any observed child frustrations. As an example, the researcher noted that Child 1 became frustrated when he was required to point to toy cars to request, but did not show frustration pointing to other items such as bubbles or balloons. The researcher recommended that the family allow free access to cars, while developing pointing with other items. Similarly, when Child 3 showed an aversion to physical prompts to point, the researcher recommended that his mother respond to reaches to initiate requesting. The researcher also addressed Parent 3's concerns that, on some days, her son wasn't in the "best mood" by explaining that adjusting expectations and strategy use on a daily basis was appropriate (e.g., creating fewer opportunities on days with lower motivation, but using play engagement strategies to look for ways to increase motivation and interest). Clinicians should collaboratively determine goals, discuss rationales, and adapt methods to meet the needs of individual children and families. Adapting 
strategies based on children's responses (e.g., engagement versus challenging behaviors) also provides a method for ensuring child assent.

\section{Limitations and Future Directions}

Although experimental control was established, there are design limitations of this study. However, these limitations may be a by-product of conducting research in lesscontrolled naturalistic contexts. First, a non-concurrent multiple probe design is not as strong as a concurrent multiple baseline design and the researchers were unable to fully randomize assignment of baseline lengths due to the fact that one participant was going to age out of early intervention. However, the primary advantage of non-concurrent designs is that they are better suited to community-based educational contexts as scheduling can present challenges for concurrent designs (Harvey et al., 2004). Similarly, while disruptions and modifications related to staff changes, and the pandemic (e.g., missing final baseline probe for Triad 3 due to COVID-19 restrictions prior to the transition to telehealth) may limit control, unexpected events and adaptations to service delivery are common in early intervention contexts. Conducting research only in ideal, controlled contexts would limit external validity. Additionally, while variability in the data could be considered a threat to internal validity, fluctuations in responding might be expected outcomes of naturalistic intervention. For instance, variability might have been affected by naturally occurring events such as a child not having a good night sleep (reported by Parent 3) or parents offering different play items. Additionally, while three target responses were selected so that parents could focus on different communicative behaviors based on the child's interests on a particular day, differences in proficiency with targets may have led to variability. For example, Child 1 initially showed higher rates of independence on days when he was more interested in items that required him to ask for assistance, as he was most proficient with the response form (handing item to parent) for this function. He did increase independent use of other targets, but rates of independent responding were influenced by daily interests. Overall, while there are control limitations of this study that were impacted by the focus on conducting research in a less-controlled community context, the consistent findings and strong effects observed provide preliminary support for the use of a brief NDBI training with early intervention providers and Latinx parents. While future research studies can consider ways to increase internal validity in natural contexts, this should not be done at the expense of external validity.

Although this study included a variety of measures related to parent, child, and provider variables, several other relevant factors were not measured. For instance, while parents were instructed to increase wait time and use lower-level prompts as children became more proficient with target behaviors, the researchers did not establish an objective way of measuring the appropriate application of this technique. Anecdotally, it appeared that Parent 3 frequently began with prompts rather than using wait time and Provider 3 did not provide feedback on wait time. Future studies could attempt to operationalize appropriate use of wait time and prompt fading, and provide both parents and providers a more concrete decision-making process. With regard to children's outcomes, this study did not examine long-term maintenance or effects on vocal speech development. Anecdotally, Child 3 (who was reported to have some vocal approximations prior to this study), was observed to pair vocal approximations with target 
responses (e.g., saying $o$ when signing OPEN and $j u$ when pointing or reaching to juice). These outcomes could be assessed in future studies and children could be followed over time. Additional outcomes for provider behaviors could also be assessed. For instance, future studies could directly measure whether providers generalize strategies to other families (which developmental specialists in the current study selfreported). Due to the COVID-19 modifications (when the types of coaching strategies that could be use changed), the researchers did not measure the overall frequency of coaching behaviors. Anecdotally, although providers 2 and 3 gave suggestions and feedback over video, provider 1 provided more regular assistance/feedback throughout in-person sessions. Although it may be beneficial for clinicians to first observe (via video or in-person) routines and then provide feedback, in-the-moment assistance or suggestions could also be advantageous in some cases. Future studies could provide clear guidelines of when to coach during a session. For example, providers could give suggestions in the moment if a parent appeared to struggle with applying a strategy or had not created an opportunity in a long time. At the end of the session, providers could use a checklist to mark strategies already addressed, and provide feedback or praise for other strategies. Less distracting methods for telehealth (e.g., bug-in-ear devices) could also be further explored.

Another potential criticism of this study is the use of the sign for MORE as a target for two children. Although it has not been evaluated experimentally, it has been suggested that children with ASD who are taught MORE as a first sign may overgeneralize this response and experience communication breakdowns when they attempt to initiate first requests with MORE (Vail \& Forbes, 2016). In this study, MORE was a target because it had already been introduced to children 2 and 3. The researcher discussed issues with over-generalization with both triads, and reiterated that each child's pointing or reaching responses should be used to request items the first time or when making choices, and MORE should only be used when the child desired more of an action, or had already initially requested an item (e.g., with a point) and wanted additional pieces. Future research could, however, explore whether teaching MORE first effects the development of additional signs. Additionally, although parents 2 and 3 indicated a desire to continue using signs (and both child 2 and 3 were observed to imitate the sign MORE at least once during baseline), there was no assessment of children's modality preference or proficiency (e.g., comparing signs or gestures to aided augmentative or alternative communication systems). Children's assent was, however, considered as parents were provided with modifications when children showed aversions to being prompted to use a specific sign or gesture.

Lastly, caution must be taken when attempting to generalize results. The study included a small number of Latinx participants, and all child participants were male. Although similar methods appeared to be effective with a female participant during the pilot, replication is required. Study methods could also be replicated across rural families, families from other underrepresented groups (e.g., Native Americans), and across Spanish-speaking Latinx families. Families in this study were required to be proficient in English primarily because the lead researcher is not fluent in Spanish, and the cooperating agency has a limited number of bilingual providers. Parent 1 was bilingual and expressed that he and his wife often spoke Spanish to their child. The researcher encouraged Parent 1 to provide vocal models in both English and Spanish, but he was primarily observed to use 
English during sessions. It is possible his tendency to speak in English was influenced by the fact that neither the developmental specialist nor researcher were fluent Spanish speakers. As shortages of trained bilingual specialists is a real-world concern in early intervention, future studies could explore whether trainings involving bilingual providers are effective for bilingual or monolingual Spanish-speaking families. Alternatively, research could explore barriers to bilingual service providers seeking additional, specialized certifications.

\section{Conclusions}

This preliminary study provides support for brief training plus coaching in NDBI strategies for parents and early intervention providers working with children with signs of ASD. Methods could be adapted to meet the needs of under-resourced early intervention programs. Additional research should seek to extend findings across a range of participants and outcomes, and efforts should be made to increase Latinx parents' access to highly-trained clinicians.

Supplementary Information The online version contains supplementary material available at https://doi.org/ 10.1007/s10882-021-09794-w.

Acknowledgements The second author was an undergraduate student at the University of New Mexico when data collection for this study occurred. We would like to thank Sophie Wong, Susanna Cole, Erin Stone, Ann Jaramillo, and Erin Gallegos for volunteering their time to assist with this project.

Funding This study was funded by the Transdisciplinary Research, Equity and Engagement Center for Advancing Behavioral Health (TREE Center) NIMHD Grant \# U54 MD004811-09.

\section{Declarations}

Ethical Approval This study was approved by a university institutional review board and all performed procedures of the study have been carried out in accordance with Helsinki Declaration (1964) and its later amendments or comparable ethical standards.

Informed Consent Informed consent was obtained for all the recruited participants. Legal representatives (i.e., parents) gave consent for child participation.

Conflict of Interest The authors declared no conflicts of interest with respect to the research, authorship, and/or publication of the article. The authors alone are responsible for the content and the writing of the article.

\section{References}

Ashburner, J., Vickerstaff, S., Beetge, J., \& Copley, J. (2016). Remote versus face-to-face delivery of early intervention programs for children with autism spectrum disorders: Perceptions of rural families and service providers. Research in Autism Spectrum Disorders, 23, 1-14. https://doi.org/10.1016/j.rasd.2015. 11.011 .

Author et al. (2018) Blinded reference. 
Buzhardt, J., Rusinko, L., Heitzman-Powell, L., Trevino-Maack, S., \& McGrath, A. (2016). Exploratory evaluation and initial adaptation of a parent training program for Hispanic families of children with autism. Family Process, 55(1), 107-122. https://doi.org/10.1111/famp.12146.

DuBay, M., Watson, L. R., \& Zhang, W. (2018). In search of culturally appropriate autism interventions: Perspectives of Latino caregivers. Journal of Autism and Developmental Disorders, 48(5), 1623-1639. https://doi.org/10.1007/s10803-017-3394-8.

Dubin, A. H., \& Lieberman-Betz, R. G. (2020). Naturalistic interventions to improve prelinguistic communication for children with autism spectrum disorder: A systematic review. Review Journal of Autism and Developmental Disorders, 7(2), 151-167. https://doi.org/10.1007/s40489-019-00184-9.

Friedman, M., Woods, J., \& Salisbury, C. (2012). Caregiver coaching strategies for early intervention providers. Infants and Young Children, 25(1), 62-82. https://doi.org/10.1097/iyc.0b013e31823d8f12.

Harvey, M. T., May, M. E., \& Kennedy, C. H. (2004). Nonconcurrent multiple baseline designs and the evaluation of educational systems. Journal of Behavioral Education, 13(4), 267-276. https://doi.org/10. 1023/b:jobe.0000044735.51022.5d.

Kratochwill, T. R., Hitchcock, J. H., Horner, R. H., Levin, J. R., Odom, S. L., Rindskopf, D. M., \& Shadish, W. R. (2013). Single-case intervention research design standards. Remedial and Special Education, 34(1), 26-38.

Leko, M. M. (2014). The value of qualitative methods in social validity research. Remedial and Special Education, 35(5), 275-286. https://doi.org/10.1177/0741932514524002.

Maenner, M. J., Shaw, K. A., \& Baio, J. (2020). Prevalence of autism spectrum disorder among children aged 8 years - autism and developmental disabilities monitoring network, 11 sites, United States, 2016. MMWR Surveillance Summaries, 69(4), 1-12. https://oi.org/10.15585/mmwr.ss6904a1.

Magaña, S., Lopez, K., Aguinaga, A., \& Morton, H. (2013). Access to diagnosis and treatment services among Latino children with autism spectrum disorders. Intellectual and Developmental Disabilities, 51(3), 141153. https://doi.org/10.1352/1934-9556-51.3.141.

Magaña, S., Parish, S. L., \& Son, E. (2016). Functional severity and Latino ethnicity in specialty services for children with autism spectrum disorder. Journal of Intellectual Disability Research, 60(5), 424-434. https://doi.org/10.1111/jir.12293.

Meadan, H., Chung, M. Y., Sands, M. M., \& Snodgrass, M. R. (2020). The cascading coaching model for supporting service providers, caregivers, and children. The Journal of Special Education, 54(2), 113-125. https://doi.org/10.1177/0022466919884070.

Medeiros, K., \& Winsler, A. (2014). Parent-child gesture use during problem solving in autistic spectrum disorder. Journal of Autism and Developmental Disorders, 44(8), 1946-1958. https://doi.org/10.1007/ s10803-014-2069-y.

Office of Special Education Programs (2020). NM Part C FFY 2018 state performance plan /annual performance report. Retrieved October 23, 2020, from https://sites.ed.gov/idea/idea-files/2020-spp-aprand-state-determination-letters-part-c-new-mexico/

Ozçalıșkan, Ș., Adamson, L. B., \& Dimitrova, N. (2016). Early deictic but not other gestures predict later vocabulary in both typical development and autism. Autism, 20(6), 754-763. https://doi.org/10.1177/ 1362361315605921.

Parker, R. I., \& Vannest, K. (2009). An improved effect size for single-case research: Nonoverlap of all pairs. Behavior Therapy, 40(4), 357-367. https://doi.org/10.1016/j.beth.2008.10.006.

Parker, R. I., Vannest, K. J., \& Davis, J. L. (2011). Effect size in single-case research: A review of nine nonoverlap techniques. Behavior Modification, 35(4), 303-322. https://doi.org/10.1177/ 0145445511399147.

Pepperdine, C. R., \& McCrimmon, A. W. (2018). Test review: Vineland adaptive behavior scales, third edition (Vineland-3) by Sparrow, S. S., Cicchetti, D. V., and Saulnier, C. A. Canadian Journal of School Psychology, 33(2), 157-163. https://doi.org/10.1177/0829573517733845.

Reszka, S. S., Boyd, B. A., McBee, M., Hume, K. A., \& Odom, S. L. (2014). Brief report: Concurrent validity of autism symptom severity measures. Journal of Autism and Developmental Disorders, 44(2), 466-470. https://doi.org/10.1007/s10803-013-1879-7.

Robins, D. L., Casagrande, K., Barton, M., Chen, C. M. A., Dumont-Mathieu, T., \& Fein, D. (2014). Validation of the modified checklist for autism in toddlers, revised with follow-up (M-CHAT-R/F). Pediatrics, 133(1), 37-45. https://doi.org/10.1542/peds.2013-1813.

Rose, V., Trembath, D., Keen, D., \& Paynter, J. (2016). The proportion of minimally verbal children with autism spectrum disorder in a community-based early intervention programme. Journal of Intellectual Disability Research, 60(5), 464-477. https://doi.org/10.1111/jir.12284.

Rowland, C. (2011). Using the communication matrix to assess expressive skills in early communicators. Communication Disorders Quarterly, 32(3), 190-201. https://doi.org/10.1177/1525740110394651. 
Sandbank, M., Bottema-Beutel, K., Crowley, S., Cassidy, M., Feldman, J. I., Canihuante, M., \& Woynaroski, T. (2020a). Intervention effects on language in children with autism: A project AIM meta-analysis. Journal of Speech, Language, and Hearing Research, 63(5), 1537-1560. https://doi.org/10.1037/ bu10000215.supp.

Sandbank, M., Bottema-Beutel, K., Crowley, S., Cassidy, M., Dunham, K., Feldman, J. I., Crank, J., Albarran, S. A., Raj, S., Mahbub, P., \& Woynaroski, T. G. (2020b). Project AIM: Autism intervention metaanalysis for studies of young children. Psychological Bulletin, 146(1), 1-29. https://doi.org/10.1037/ bul0000215.

Schopler, E., Reichle, R. J., and Renner, B. R. (2010). Childhood autism rating scale (2nd ed.). Western Psychological Services.

Schreibman, L., Dawson, G., Stahmer, A. C., Landa, R., Rogers, S. J., McGee, G. G., et al. (2015). Naturalistic developmental behavioral interventions: Empirically validated treatments for autism spectrum disorder. Journal of Autism and Developmental Disorders, 45(8), 2411-2428. https://doi.org/10.1007/ s10803-015-2407-8.

Shumway, S., \& Wetherby, A. M. (2009). Communicative acts of children with autism spectrum disorders in the second year of life. Journal of Speech, Language, and Hearing Research, 52(5), 1139-1156. https:// doi.org/10.1044/1092-4388(2009/07-0280).

Sparrow, S. S., Cicchetti, D. V., and Saulnier, C. A. (2016). Vineland adaptive behavior scales, (3rd ed.) Pearson.

Sutherland, R., Trembath, D., \& Roberts, J. (2018). Telehealth and autism: A systematic search and review of the literature. International Journal of Speech-Language Pathology, 20(3), 324-336. https://doi.org/10. 1080/17549507.2018.1465123.

Swanson, M. R., Donovan, K., Paterson, S., Wolff, J. J., Parish-Morris, J., et al. (2019). Early language exposure supports later language skills in infants with and without autism. Autism Research, 12(12), 1784-1795. https://doi.org/10.1002/aur.2163.

Trembath, D., Gurm, M., Scheerer, N. E., Trevisan, D. A., Paynter, J., Bohadana, G., Roberts, J., \& Iarocci, G. (2019). Systematic review of factors that may influence the outcomes and generalizability of parentmediated interventions for young children with autism spectrum disorder. Autism Research, 12(9), 1304 1321. https://doi.org/10.1002/aur.2168.

United States Department of Education (2020). IDEA section 618 data products: Static tables. Retrieved October 23, 2020, from https:/www2.ed.gov/programs/osepidea/618-data/static-tables/index.html

Vail, T., and Forbes, H. (2016). No more "More": A response to "in defense of more." Aspire early intervention. Retrieved October 23, 2020, from https://www.aspireearlyintervention.com/news/10reasons-i-still-teach-the-sign-more-first/

Watson, P. J., \& Workman, E. A. (1981). The non-concurrent multiple baseline across- individuals design: An extension of the traditional multiple baseline design. Journal of Behavior Therapy and Experimental Psychiatry, 12(3), 257-259. https://doi.org/10.1016/0005-7916(81)90055-0.

Zwaigenbaum, L., Bauman, M. L., Choueiri, R., Kasari, C., Carter, A., Granpeesheh, D., et al. (2015). Early intervention for children with autism spectrum disorder under 3 years of age: Recommendations for practice and research. Pediactrics, 136(Supplement), S60-S81. https://doi.org/10.1542/peds.2014-3667e.

Publisher's Note Springer Nature remains neutral with regard to jurisdictional claims in published maps and institutional affiliations.

\section{Affiliations}

\section{Cindy Gevarter ${ }^{1}$ - Adriana Medina Najar ${ }^{2}$ - Jennifer Flake ${ }^{1} \cdot$ Felicia Tapia-Alvidrez ${ }^{3}$ - Alixandria Lucero ${ }^{3}$}

1 Department of Speech and Hearing Sciences, The University of New Mexico, 1700 Lomas NE, MSC01 1195, 1 University of New Mexico, Albuquerque, NM 87131, USA

2 Southern Connecticut State University, New Haven, CT 06515, USA

3 PB\&J Family Services Inc, Albuquerque, NM 87105, USA 